\title{
What do patients take into account when they choose their dentist? Implications for quality improvement
}

\author{
Marius-Ionuț Ungureanu' ${ }^{1,2}$ \\ Floarea Mocean' \\ 'Department of Public Health \\ and Management, luliu Hațieganu \\ University of Medicine and Pharmacy, \\ ${ }^{2}$ Center for Health Policy and \\ Public Health, College of Political, \\ Administrative and Communication \\ Sciences, Babeș-Bolyai University, \\ Cluj-Napoca, Romania
}

This article was published in the following Dove Press journal:

Patient Preference and Adherence

27 November 2015

Number of times this article has been viewed

\begin{abstract}
Background: From dental care providers' perspective, it is vital to put efforts into improving the quality of services delivered. Therefore, the exploration of patients' satisfaction with dental services and their preferences with regard to the factors involved in their dental care choices should be one of the main areas of focus in a dental care office, by focusing on the quality management.
\end{abstract}

Aims: The aim of this research was to determine the factors that patients consider important when choosing a dentist, and bring evidence on the importance of implementing a quality management system for dental care.

Methods and participants: The data analysis was conducted on a national representative sample of patients who had visited a dentist at least once in the last 12 months, in Romania. Using a survey which explored patients' satisfaction with their dental care visits, data were collected between October and November 2012 with the help of computer-assisted telephone interviews. Information about socio-demographics, frequency of accessing dental care, factors associated with choosing a dentist, and the level of patients' satisfaction were collected.

Results: Out of the 1,650 people who agreed to participate in the study, 724 respondents (43.8\%) represented the sample who visited a dentist at least once in the last 12 months and whose responses were analyzed in this article. The majority of the respondents were satisfied with the quality of dental services received, with $85.91 \%$ of them rating their satisfaction with 5 , on a scale from 1 to 5 . According to a high majority of the patients ( $n=680,93.92 \%)$, the communication with their dentist was extremely easy. The most frequently mentioned factors that respondents took into account when choosing a dentist were the dentist's competence $(22.22 \%)$, the recommendation from someone known (20.56\%), and the overall quality of the service provided (19.72\%). Among the other factors mentioned were patience and respect.

Conclusion: The factors patients consider important when choosing a dentist are the dentist's competence, the recommendation from someone known, and the overall quality of the service provided, but interpersonal factors such as patience and respect are also valued. Our results are relevant for dental education providers, who should consider shaping their curricula as to ensure they train future dentists in a manner that will meet patients' expectations and increase patient satisfaction levels.

Keywords: patient preference, dental care, quality improvement, patient satisfaction

Correspondence: Marius-lonuț Ungureanu

Department of Public Health and Management, luliu Hațieganu University of Medicine and Pharmacy, 8 Victor Babeș Street, Cluj-Napoca 400008, Romania $\mathrm{Tel}+40264597256$

Email m.i.ungureanu@gmail.com

\section{Introduction}

\section{The importance of characteristics influencing patients' choice of a dentist}

Various research have shown that patients choose their dentist based on a variety of factors. ${ }^{1,2}$ These factors can be divided into factors related to dentists only (ability 
to communicate, express empathy, and manage pain), factors related to the dental office where the dentists are providing the dental services (geographical situation and cleanliness), as well as to the characteristics of the service delivered (timeliness, correspondence with the latest scientific achievements). ${ }^{3,4}$ Other studies have suggested that any similarities between specific characteristics of the patient and dentist (such as sex or race) can also be involved in patients' choice of a dentist. ${ }^{5-7}$

\section{The importance of quality management approaches in dentistry}

Given the impact that quality has on the service receivers, it became a highly debated matter, as well as quality management, which proves to be one of the greatest challenges in the field of dentistry. ${ }^{8}$ However, despite the challenges, quality management has a significant role in assuring the delivery of quality dental care services. ${ }^{9}$

The structure, the processes, and the outcomes an organization has, account for defining and measuring quality. Although quality management processes have been developed for health care, they were translated to dental care practices to increase and improve quality assurance. ${ }^{10}$

According to the Institute of Medicine, services are of quality, when they are safe, effective, patient centered, timely, efficient, and equitable. Nonetheless, even if this is not the only definition provided for quality, it is widely accepted that the quality is multidimensional and its core underlying concepts are patient safety, patient experience, access, and clinical effectiveness. ${ }^{11}$

One of the ways of applying a quality management system is by adopting the ISO 9000 family of standards, which include a set of guidelines and standards on good quality management practices. ${ }^{12}$ The ISO 9000 series comprises five international standards on quality assurance and quality management, focusing on health care facilities. Although receiving some criticism, ISO standards are adopted in many countries, mostly in health care and in a smaller proportion in dental care, in countries such as Sweden, the UK, and Canada, ${ }^{12-14}$ and are considered core measures of quality management in different private settings.

The first principle of the ISO 9000 standards, customer focus, takes into consideration the importance of clients pose for a dental practice and leads toward the improvement of patient satisfaction. The leadership of the dental practice refers to offering a purpose and a direction, as well as an environment in which all stakeholders can work toward achieving the objectives of the dental care unit. The focus on people's involvement emphasizes the fact that the personnel, regardless of the position, represent the essence of the unit and their abilities should be used to benefit the organization. This process motivates employees, makes them become more accountable for their performance, and makes them interested in participating at the continuous improvement of the dental care unit. ${ }^{11}$

According to the principle of continuous improvement, the overall performance should be a permanent objective of the organization. The benefits of applying this principle are a performance advantage and an alignment of improved activities at all levels of the dental practice. This leads to the need of training the personnel in the methods of continual improvement, to establishing goals and measurements and acknowledging improvement. ${ }^{11}$

\section{Aim of the paper}

The aim of the research was to determine the factors that patients consider important when choosing a dentist in Romania, and bring evidence on the importance of implementing a quality management system for dental care. We also explored the frequency of dental visits and their association with patients' socio-demographic characteristics.

\section{Methods}

\section{Study design}

This study has been approved by the Research Ethics Committee of the Iuliu Haţieganu University of Medicine and Pharmacy. Informed consents have been obtained from all participants in the study, prior to their enrollment. This study had a cross-sectional design and was part of an ongoing national funded research grant titled "Development of a novel Quality Management System for Use in Dental Practice", funded by the National Authority for Scientific Research, CNCS-UEFISCDI, project number PN-IIPCCA2011-3.1-1208, and implemented at a national level. This research is withdrawn from the first part of the formative research component, focusing on exploring dental service users' attitudes and perceptions on the quality of services received. The data were collected from a representative sample of 1,650 people in Romania, using computer-assisted telephone interviews (CATIs). Respondents, aged 18 and above, were asked whether they visited a dentist in the last 12 months prior to the CATIs. Out of the 1,650 respondents, a sample of $724(43.8 \%)$ did visit a dentist in the 12 months prior to the CATIs, and represented the sample of this research.

CATIs were used as a data collection tool due to its survey-based, rapid, flexible, and cost-effective characteristics in public health data collection. A computerized 
questionnaire was used to capture respondents' feedback on the dental care services they have received during the made to the dentist in the last 12 months. The computerized questionnaire was split in three parts sections looking at respondents' socio-demographic characteristics, details of dental services used and a self-assessment of the quality of services (focusing on comfort, costs, and communication). A Likert Scale was used to grade the items included in the self-assessment of the quality of services.

For the purpose of this study, the following variables were of interest: the respondents' preference for dentistrelated factors, the overall satisfaction with their last dental visit, satisfaction related to the clinical aspects of care, as well aspects connected to the environment where they have received the care. Statistical Package for Social Sciences (SPSS) version 17.0 has been employed for the statistical analyses. We used frequencies for the description of the socio-demographic characteristics of the respondents. We also used chi-square $\left(\chi^{2}\right)$ to test associations between various categorical variables.

\section{Results}

Among the 724 patients who visited a dental care unit in the last 12 months, $62.57 \%$ were females $(n=453)$ and $37.73 \%$ were males $(\mathrm{n}=271)$ (Table 1). Residents of urban areas represented the majority of our sample $(\mathrm{n}=522,72.10 \%)$, as compared to the residents of rural areas $(n=202,27.90 \%)$. The mean age of participants was 46.56 years. The best represented age groups was 45-49 years, the working adult population, comprising $28.73 \%(\mathrm{n}=208)$ of the total respondents, followed by adults older than 60 years of age $(\mathrm{n}=187,25.83 \%)$, and young adults $(\mathrm{n}=176,24.31 \%)$. Youth represented $21.13 \%$ of the total respondents. Most of the participants in the study had attained medium education $(\mathrm{n}=446,61.60 \%)$.

When respondents have been asked whether they have a dentist that they visit regularly, $78.18 \%(\mathrm{n}=566)$ replied affirmatively. However, a large proportion of respondents admitted that they visit their dentist only when they experience toothache (27.85\%).

At the end of their most recent dental visit, only $39.64 \%$ of our respondents have been asked by their dentist whether they have been satisfied with their visit. The dental facility most recently visited has been a private one for $85.36 \%$ of our respondents.

We analyzed the data to test if there are any associations between the frequency of dental visits and respondents' socio-demographic characteristics - sex, age, residence area type, and education (Table 2). The characteristics for which
Table I Socio-demographic description of participants

\begin{tabular}{|c|c|}
\hline Characteristic & $\mathbf{N}(\%)$ \\
\hline \multicolumn{2}{|l|}{$\operatorname{Sex}(N=724)$} \\
\hline Male & $271(37.73)$ \\
\hline Female & $453(62.57)$ \\
\hline \multicolumn{2}{|l|}{ Residence area $(\mathrm{N}=724)$} \\
\hline Urban & $522(72.10)$ \\
\hline Rural & $202(27.90)$ \\
\hline Age (mean $\pm S D ; N=724)$ & $46.56 \pm 17.11$ \\
\hline \multicolumn{2}{|l|}{ Age $(N=724)$ (years) } \\
\hline $18-29$ & $153(21.13)$ \\
\hline $30-44$ & I $76(24.3 \mid)$ \\
\hline $45-59$ & $208(28.73)$ \\
\hline $60+$ & $187(25.83)$ \\
\hline \multicolumn{2}{|c|}{ Highest education level attained ( $\mathrm{N}=724)$} \\
\hline Primary education & $66(9.12)$ \\
\hline Medium & $446(61.60)$ \\
\hline Higher education & $212(29.28)$ \\
\hline \multicolumn{2}{|c|}{ Existence of a dentist who is regularly visited $(\mathrm{N}=724)$} \\
\hline Yes & $566(78.18)$ \\
\hline No & $158(21.82)$ \\
\hline \multicolumn{2}{|c|}{ Usual frequency of dental care visits $(\mathrm{N}=7 \mid 8)$} \\
\hline Once every 4 months & $91(12.67)$ \\
\hline Once every 6 months & $177(24.66)$ \\
\hline Once a year & $195(27.16)$ \\
\hline At an interval $>I$ year & $55(7.66)$ \\
\hline Only when I have tooth ache & $200(27.85)$ \\
\hline \multicolumn{2}{|c|}{$\begin{array}{l}\text { At the end of the dental visit, have you been asked by the dentist } \\
\text { whether you have been satisfied with your visit? }\end{array}$} \\
\hline Yes & $428(59.12)$ \\
\hline No & $287(39.64)$ \\
\hline I do not remember & $9(1.24)$ \\
\hline \multicolumn{2}{|c|}{ At your last dental visit, did you go to a public or private facility? } \\
\hline Private & $618(85.36)$ \\
\hline Public & $94(12.98)$ \\
\hline I do not remember & $12(1.66)$ \\
\hline
\end{tabular}

Abbreviation: SD, standard deviation.

the association was found to be statistically significant were age $(P=0.006)$, the residence area type $(P=0.001)$, and the highest level of education attained $(P<0.001)$.

When respondents have been asked about the factors that they considered when they chose their dentist, a variety of factors were mentioned regarding the factors who determine choosing a dentist. Factors related to dentists' professional skills $(22.22 \%)$, personal traits, and the characteristics of the facility were mentioned (Table 3 ). The most frequently mentioned three factors were: the dentist's professional skills ( $22.22 \%$ ), being recommended by someone they knew $(20.56 \%)$ and the overall quality of the dental care services provided (19.72\%). Among other factors mentioned were: dentist's patience $(9.44 \%)$, the distance from home to dental office ( $8.61 \%)$, dentist's respect for patients $(6.39 \%)$, and the degree to which schedules are respected $(1.80 \%)$.

A $\chi^{2}$ test for independence indicated no significant association between sex and the type of facility visited, 
Table 2 The distribution of respondents' visit to the dentist according to respondents' socio-demographic characteristics

\begin{tabular}{|c|c|c|c|c|c|c|c|}
\hline \multirow{3}{*}{$\begin{array}{l}\text { Socio-demographic } \\
\text { characteristic }\end{array}$} & \multicolumn{7}{|c|}{ Frequency of visits to the dentist's office } \\
\hline & \multirow{2}{*}{$\begin{array}{l}\text { Once every } \\
4 \text { months } \\
N(\%)\end{array}$} & \multirow{2}{*}{$\begin{array}{l}\text { Once every } \\
6 \text { months } \\
N(\%)\end{array}$} & \multirow{2}{*}{$\begin{array}{l}\begin{array}{l}\text { Once a } \\
\text { year }\end{array} \\
\mathbf{N}(\%)\end{array}$} & \multirow{2}{*}{$\begin{array}{l}\text { At an interval }>\mathbf{I} \\
\text { year } \\
\mathbf{N}(\%)\end{array}$} & \multirow{2}{*}{$\begin{array}{l}\text { Only when I } \\
\text { have tooth ache } \\
\mathbf{N}(\%)\end{array}$} & \multirow[t]{2}{*}{$\chi^{2}$} & \multirow[t]{2}{*}{$P$-value } \\
\hline & & & & & & & \\
\hline \multicolumn{8}{|l|}{ Sex } \\
\hline Male & $39(42.86)$ & $63(35.60)$ & $68(34.87)$ & $25(45.45)$ & $72(36)$ & 5.82 & 0.324 \\
\hline Female & $52(57.14)$ & $114(64.40)$ & $127(65.13)$ & $30(54.55)$ & $128(64)$ & & \\
\hline Total & 91 & 177 & 195 & 55 & 200 & & \\
\hline \multicolumn{8}{|l|}{ Age (years) } \\
\hline $18-29$ & $25(27.47)$ & $5 \mathrm{I}(28.8 \mathrm{I})$ & $36(18.46)$ & $7(12.73)$ & $33(16.5)$ & 32.05 & 0.006 \\
\hline $30-44$ & $28(30.77)$ & $49(27.68)$ & $47(24.10)$ & $12(21.82)$ & $40(20)$ & & \\
\hline $45-59$ & $23(25.27)$ & $37(20.90)$ & $64(32.82)$ & $19(34.54)$ & $62(31)$ & & \\
\hline Older than 60 & $15(16.49)$ & $40(22.61)$ & $48(24.62)$ & $17(30.91)$ & $65(32.5)$ & & \\
\hline Total & 91 & 177 & 195 & 55 & 200 & & \\
\hline \multicolumn{8}{|l|}{ Residence area type } \\
\hline Urban & $73(80.22)$ & I43 (80.79) & I 40 (7I.79) & $35(63.64)$ & $129(64.5)$ & 21.82 & 0.001 \\
\hline Rural & $18(19.78)$ & $34(19.21)$ & $55(28.21)$ & $20(36.36)$ & 7I (35.5) & & \\
\hline Total & 91 & 177 & 195 & 55 & 200 & & \\
\hline \multicolumn{8}{|l|}{ Education } \\
\hline Primary education & $4(4.40)$ & $12(6.78)$ & $20(10.25)$ & $8(14.54)$ & $22(\mathrm{II})$ & 34.59 & $<0.001$ \\
\hline Medium & $57(62.63)$ & $89(50.28)$ & $12 \mid(62.05)$ & $34(61.82)$ & $139(69.5)$ & & \\
\hline Higher education & $30(32.97)$ & $76(42.94)$ & $54(27.70)$ & $13(23.64)$ & $39(19.50)$ & & \\
\hline Total & 91 & 177 & 195 & 55 & 200 & & \\
\hline
\end{tabular}

$P=0.143, \pi=0.09$. A $\chi^{2}$ test for independence indicated no significant association between sex and whether the respondent has been asked by the dentist if he/she was satisfied, $P=0.102, \pi=0.07$. A $\chi^{2}$ test for independence indicated no significant association between sex and whether the respondent has a dentist that they visit regularly, $\chi^{2}=5.82$, $P=0.069, \pi=0.07$.

However, a $\chi^{2}$ test for independence indicated a significant association between the respondents' residence area type and whether they have a dentist that they visit regularly, $P=0.001, \pi=0.12$.

The assessment of overall satisfaction, as well as specific areas of dental care rendered high levels of satisfaction

Table 3 The factor that was most important in choosing a dentist $(\mathrm{N}=720)$

\begin{tabular}{ll}
\hline Factor & N (\%) \\
\hline Dentist's competence & I60 (22.22) \\
Recommendation made by someone I know & I $48(20.56)$ \\
Overall quality of the service provided & $142(19.72)$ \\
Dentist's patience & $68(9.44)$ \\
Distance from home to dental office & $62(8.61)$ \\
Dentist's respect for patients & $46(6.39)$ \\
The quality of equipment in the dentist's office & $34(4.73)$ \\
Dentist's notoriety & $32(4.44)$ \\
The degree to which schedules are respected & $13(1.80)$ \\
Overall aspect of the dentist's office & $\mathrm{II}(1.53)$ \\
Other (unspecified) & $4(5.56)$ \\
\hline
\end{tabular}

among the study respondents. When asked about the overall satisfaction, $85.91 \%$ of respondents answered that it was very high (Table 4). Respondents' level of satisfaction about the thoroughness of explanations received from their dentist has been higher in $81.89 \%$ of the cases. Moreover, $93.92 \%$ of the respondents highly agreed that their communication with the dentist has been easy.

\section{Discussion}

The participants in our study seem to value different factors when they choose a dentist. Unsurprisingly, the most frequently mentioned is the dentists' competence (22.22\%), similar to other studies conducted previously. ${ }^{15}$ However, the second factor is the recommendation made by someone that they know (20.56\%). This result is a confirmation of the importance that word-of-mouth still represents a strong marketing mechanism for dental services promotion. Other studies have found that the availability of different treatments and a friendly environment are important decision factors. ${ }^{15}$

Our results also suggest that participants in the study perceive a difference between what is circulated by a certain dentist, as compared to an advice received from a known and trusted person. According to our results, a dentist's reputation is placed the eighth on a list of factors that our participants have considered (with 4.44\%), as compared to the second place held by a recommendation by someone they know (with 
Table 4 Participants' satisfaction with the dental services received

\begin{tabular}{|c|c|c|c|c|c|}
\hline Service characteristic & Very low & Low & Medium & High & Very high \\
\hline \multicolumn{6}{|l|}{ Overall satisfaction $(\mathrm{N}=724)$} \\
\hline$N(\%)$ & II (I.52) & $10(1.38)$ & $10(1.38)$ & $7 \mathrm{I}(9.8 \mathrm{I})$ & $622(85.91)$ \\
\hline \multicolumn{6}{|c|}{ More information expected $\left(\mathrm{N}=7 \mid 4^{*}\right)$} \\
\hline$N(\%)$ & $328(4.59)$ & $59(8.26)$ & $41(5.74)$ & $88(12.32)$ & $198(27.73)$ \\
\hline \multicolumn{6}{|c|}{ Thorough explanations received from dentist ( $\left.\mathrm{N}=723^{* *}\right)$} \\
\hline$N(\%)$ & $27(3.73)$ & $19(2.62)$ & $17(2.35)$ & $68(9.4 I)$ & $592(81.89)$ \\
\hline \multicolumn{6}{|c|}{ Communication with the dentist was easy $(\mathrm{N}=724)$} \\
\hline $\mathrm{N}(\%)$ & $2(0.28)$ & $2(0.28)$ & $2(0.28)$ & $38(5.25)$ & $680(93.92)$ \\
\hline
\end{tabular}

Notes: *Missing ten cases. **Missing one case.

$20.56 \%)$. Whereas these results might have no influence on how a dentist is marketing his or her practice, they show that the perception that a patient has on the quality of dental services delivered can either increase or decrease the number of patients who will visit that doctor.

The fact that the factors mentioned by participants are so different has tremendous implications for the quality assurance process in the dental office. First, it stresses the importance of dentists' development of inter-personal and communication skills. This finding is particularly relevant for the providers of dental education, urging them to include classes on communication, leadership, and negotiation skills in their curricula, which proved to be successful in other settings. ${ }^{16,17}$ Second, it highlights the importance of creating a workplace environment focused on a shared effort to attain the patient's highest level of satisfaction. Recent studies have shown that, there is an association between dentists' satisfaction of the working environment and patients' dental care service satisfaction. ${ }^{18}$

According to our results, there is an association $(P<0.05)$ between the frequency of dental visits and age $(P=0.006)$, residence area type $(P=0.001)$, and the highest level of education attained $(P<0.001)$. These results are similar to the findings reported by other studies. ${ }^{19}$ The implications of these results can be managed at different levels. At a dental office level, the relevance is with regard to dentists' efforts to educate their patients about the importance of regular dental visits. The high proportion of respondents who visit a dentist only when they experience toothache is a concern, since, most often, in these situations dentists have to take a more radical approach to treatment. Other studies show that a higher frequency of dental visits (at least every 6 months) leads to better prevention of dental complications. ${ }^{20}$ From a dental education provider's perspective, our results should encourage a more consistent focus on including dental education classes in the curricula. Studies conducted in the USA have proven that these classes will be translated in an increased effectiveness of dentists' efforts to educate their patients about the importance of dental prevention and regular visits to the dentist. ${ }^{21}$ From a quality management perspective, our results support the idea that the involved in the implementation of quality processes at a dental office level should focus their attention on designing, implementing and evaluating the interventions aimed at increasing patients' retention at the dental office level.

\section{Limitations}

Our study has some inherent limitations. First, it presents data from the users of dental services in a system where these services are paid out-of-pocket. This could have an influence that the study was not able to capture, thus making the results not generalizable to systems where dental care is provided in the public system. To address this limitation, comparative studies need to be conducted in health care systems with different arrangement of the service financing. Second, our respondents report high levels of satisfaction about the services they received, despite the fact that a majority of them visit a dentist only they experience toothache. These results could suggest that respondents have a low health literacy level, which has not been explored in the study. To address this limitation, further studies are needed to assess respondents' health literacy levels, with a special focus on dental care. Furthermore, qualitative research is needed to explore in more depth the determinants of patients' satisfaction with dental services, as well as their expectations from the quality management of dental care providers.

\section{Conclusion}

The factors patients consider important when choosing a dentist are the dentist's competence, the recommendation from someone known and the overall quality of the service provided, but interpersonal factors such as patience and respect are also valued. Our results are relevant to the providers of dental education, who should consider shaping their curricula to ensure they train future dentists in a manner that 
will contribute to meeting patients' expectations and attaining the highest patient satisfaction levels.

\section{Acknowledgment}

This project has received funding from the National Authority for Scientific Research, CNCS-UEFISCDI, number PN-IIPCCA-2011-3.1-1208.

\section{Disclosure}

The authors report no conflicts of interest in this work.

\section{References}

1. Nash DA. Ethics, empathy, and the education of dentists. J Dent Educ. 2010;74(6):567-578.

2. Hojat M. Ten approaches for enhancing empathy in health and human services. J Health Hum Serv Adm. 2009; Spring:412-450.

3. Furnham A, Swami V. Patient preferences for dentists. Psychol Health Med. 2009;14(2):143-149.

4. Stokes J, Pack A, Spears G. A comparison of patients' perception of dental care offered by male and female dentists in New Zealand. Int Dent J. 1992;42:217-222.

5. Bender DJ. Patient preference for a racially or gender-concordant student dentist. J Dent Educ. 2007;71(6):726-745.

6. Lillie-Blanton M, Brodie M, Rowland D, Altman D, McIntosh M. Race, ethnicity, and the health care system: public perceptions and experiences. Med Care Res Rev. 2000;57(Suppl 1):218-235.

7. Stevens GD, Shi L, Cooper LA. Patient-provider racial and ethnic concordance and parent reports of the primary care experiences of children. Ann Fam Med. 2003;1(2):105-112.

8. Badri MA, Attia S, Ustadi AM. Healthcare quality and moderators of patient satisfaction: testing for causality. Int J Health Care Qual Assur. 2009;22:15-20.
9. Mills I, Batchelor P. Quality indicators: the rationale behind their use in NHS dentistry. Br Dent J. 2011;211:11-15.

10. Goetz K, Campbell SM, Broge B, Brodowski M, Wensing M, Szecsenyi J. Effectiveness of a quality management program in dental care practices. BMC Oral Health. 2014;14:41.

11. ISO. Quality Management Principles. Geneva: ISO. Available from: http://www.iso.org/iso/qmp_2012.pdf. Accessed February 2015.

12. Evans J. Quality and Performance Excelence. Mason: Cengage Learning; 2013:105-119.

13. Legido-Quigley H, McKee M, Nolte E, Glinos IA. Assuring the Quality of Healthcare in the European Union: A Case for Action. European Observatory on Health Systems and Policies. Copenhagen: WHO Regional Office for Europe; 2008.

14. Casas M, Kenny DJ, Johnston DH. Experience of 2 dental clinics registered to ISO 9002. J Can Dent Assoc. 2003;69(4):215-218.

15. Iqbal M, Jameel A, Girach MM. Factors affecting patients' choice of dental services. Pakistan Oral Dent J. 2014;34(4):691-695.

16. DePaola DP, Slavkin HC. Reforming dental health professions education: a white paper. J Dent Educ. 2004;68(11):1139-1150.

17. Roth K. Dental education: a leadership challenge for dental educators and practitioners. J Dent Educ. 2007;71(8):983-987.

18. Lee CT, Zhang S, Leung YY, Li SK, Tsang CC, Chu CH. Patients' satisfaction and prevalence of complications on surgical extraction of third molar. Patient Preference and Adherence. 2015;9:257-263.

19. Ide R, Mizoue T, Tsukiyama Y, Ikeda M, Yoshimura T. Evaluation of oral health promotion in the workplace: the effects on dental care costs and frequency of dental visits. Commun Dent Oral Epidemiol. 2001; 29(3):213-219.

20. Carrillo-Diaz M, Crego A, Armfield JM, Romero-Maroto M. Treatment experience, frequency of dental visits, and children's dental fear: a cognitive approach. Eur J Oral Sci. 2012;120(1):75-81.

21. Ronis DL, Lang WP, Farghaly MM, Passow E. Tooth brushing, flossing, and preventive dental visits by detroit-area residents in relation to demographic and socioeconomic factors. J Public Health Dent. 1993; 53(3):138-145.
Patient Preference and Adherence

\section{Publish your work in this journal}

Patient Preference and Adherence is an international, peer-reviewed, open access journal that focuses on the growing importance of patient preference and adherence throughout the therapeutic continuum. Patient satisfaction, acceptability, quality of life, compliance, persistence and their role in developing new therapeutic modalities and compounds to optimize

\section{Dovepress}

clinical outcomes for existing disease states are major areas of interest for the journal. This journal has been accepted for indexing on PubMed Central. The manuscript management system is completely online and includes a very quick and fair peer-review system, which is all easy to use. Visit http://www. dovepress.com/testimonials.php to read real quotes from published authors. 